\title{
Standsicherheitsnachweise von Gewichtsstaumauern mit CADAM2D
}

\author{
Edwin Staudacher (iD $\cdot$ Gerald Zenz
}

Online publiziert: 5 . Juni 2020

(c) Der/die Autor(en) 2020

\section{Zusammenfassung Im Talsperrenbau wird die Auslegung und Überwachung von Talsperren durch numerische Mo- delle unterstützt. Aufgrund der komple- xen geometrischen und mechanischen Zusammenhänge ist es der Stand der \\ Schlüsselwörter Talsperren . \\ Bauwerkssicherheit · Numerische Modelle $\cdot$ Gewichtsstaumauern \\ Safety assessment of concrete gravity dams using CADAM2D} Technik, dass die Finite-Elemente-Methode zum Einsatz kommt. In frühen Projektstadien oder zur überschlägigen Berechnung im Betrieb lassen sich die grundlegenden Standsicherheitsnachweise von Gewichtsstaumauern mit der frei verfügbaren Software CADAM2D unter Berücksichtigung der einschlägigen Richtlinien im Talsperrenwesen durchführen. Anhand der im englischsprachigen Raum bekannten „Gravity Method“ wird die Spannungsverteilung der Aufstandsfläche bzw. der Arbeitsfugen des Sperrenkörpers unter Berücksichtigung der Balkentheorie berechnet. Dabei kann bei Überschreiten der Zugfestigkeit auch ein Öffnen der Fugen zugelassen werden. Aufbauend auf dieser Berechnung gibt CADAM2D die Sicherheitsfaktoren wie z. B. Gleiten oder Kippen aus. Im Erdbebenlastfall werden die Trägheitskräfte durch Anwendung quasi-statischer Ansätze berechnet und in weiterer Folge mit den statischen Lasten kombiniert. Weitere Programmmodule ermöglichen probabilistische Sicherheitsberechnungen oder auch Sensitivitätsanalysen, welche die meist komplexe Beurteilung der Bauwerkssicherheit unterstützen. Die flache Lernkurve bei der Bedienung und die vielseitigen Funktionen stellen, zusammen mit der freien Verfügbarkeit des Programms, ein attraktives Paket vor allem im Ausbildungsbereich dar.

DI E. Staudacher $(\bowtie)$.

Univ.-Prof. DI Dr. G. Zenz

Institut für Wasserbau und

Wasserwirtschaft, Technische

Universität Graz, Stremayrgasse

10/II, $8010 \mathrm{Graz}$, Österreich

edwin.staudacher@tugraz.at

Univ.-Prof. DI Dr. G. Zenz

gerald.zenz@tugraz.at
Abstract In dam engineering the design and monitoring of dams is supported by numerical methods. It is state of the art to use sophisticated methods, like the finite element method, due to the complex geometric and structural relationships. In early project stages or during operation of the facilities, the preliminary structural assessment of concrete gravity dams can be carried out with the freely available software called CADAM2D, which accounts for relevant guidelines in dam engineering. Based on the so-called gravity method the stress distributions in base and lift joints is computed according to the beam theory. In case of exceedance of tensile strength, cracks may be initiated and propagated due to increase of uplift pressures. CADAM2D computes the relevant safety factors based on the stress distribution and external loads. In the event of an earthquake, inertia forces are calculated using quasi-static methods that are combined with static loads. Additional program modules feature probabilistic safety or sensitivity analyzes to help to understand and conduct the complex safety assessment of these structures. The rather flat learning curve and versatile features together with the fact that this software is freely available makes it an attractive package, especially for teaching purpose.

Keywords Dams · structural safety assessment · numerical models . concrete gravity dams

\section{Einleitung}

Die strukturelle sowie konstruktive Auslegung und Überwachung wasserbaulicher Anlagen ist durch das vorhandene
Schadenspotenzial im Einflussbereich dieser Bauwerke von besonderem Interesse. Diese Anlagen zur Erzeugung und Speicherung von elektrischer Energie können generell in zwei Gruppen eingeteilt werden: einerseits in Speicher- bzw. Pumpspeicheranlagen (Mittel- und Hochdruckanlagen) und andererseits in Laufkraftanlagen (Niederdruckanlagen).

Es ist Stand der Technik, dass die Berechnungen zur Dimensionierung bzw. Nachrechnung (z.B. aufgrund aktualisierter Richtlinien oder Normen) der bautechnischen und elektromaschinellen Anlagenteile mithilfe numerischer Methoden durchgeführt werden. Aus bautechnischer Sicht werden statische sowie dynamische Berechnungen von Krafthäusern, Wehranlagen und Talsperren unter Anwendung der Finite-Elemente-Methode (FE-Methode) durchgeführt, um die strukturelle Sicherheit beurteilen und nachweisen zu können. Im Bereich der Talsperren erfolgt diese Beurteilung prinzipiell unter Berücksichtigung des gegenseitigen Einflusses der Struktur mit dem Untergrund, dem aufgestauten Wasser im Stausee und weiteren Umwelteinflüssen. Talsperren aus Beton lassen sich in Bogenstaumauern und Gewichtsmauern unterteilen, wobei heutzutage vor allem erstere in den Planungsphasen, angefangen vom Entwurf bis zur Nachweisführung von Bestandsbauten, aufgrund der Komplexität mit der FEMethode untersucht werden.

Die Komplexität der Modellierung hängt von der Geometrie und dem Detaillierungsgrad der Diskretisierung, der Art der Analyse (statisch, dynamisch), aber auch von der Wahl des Materialmodells (linear oder nichtlinear) bzw. vom Einsatz struktureller Nichtlinearitäten ab (Staudacher und Zenz 2017, 2019). Das Technical Committee on Numerical Analysis and Design of Dams der International Commission On Large Dams (ICOLD) veranstaltet in regelmäßigen Abständen sogenannte Benchmark-Workshops, in denen die 


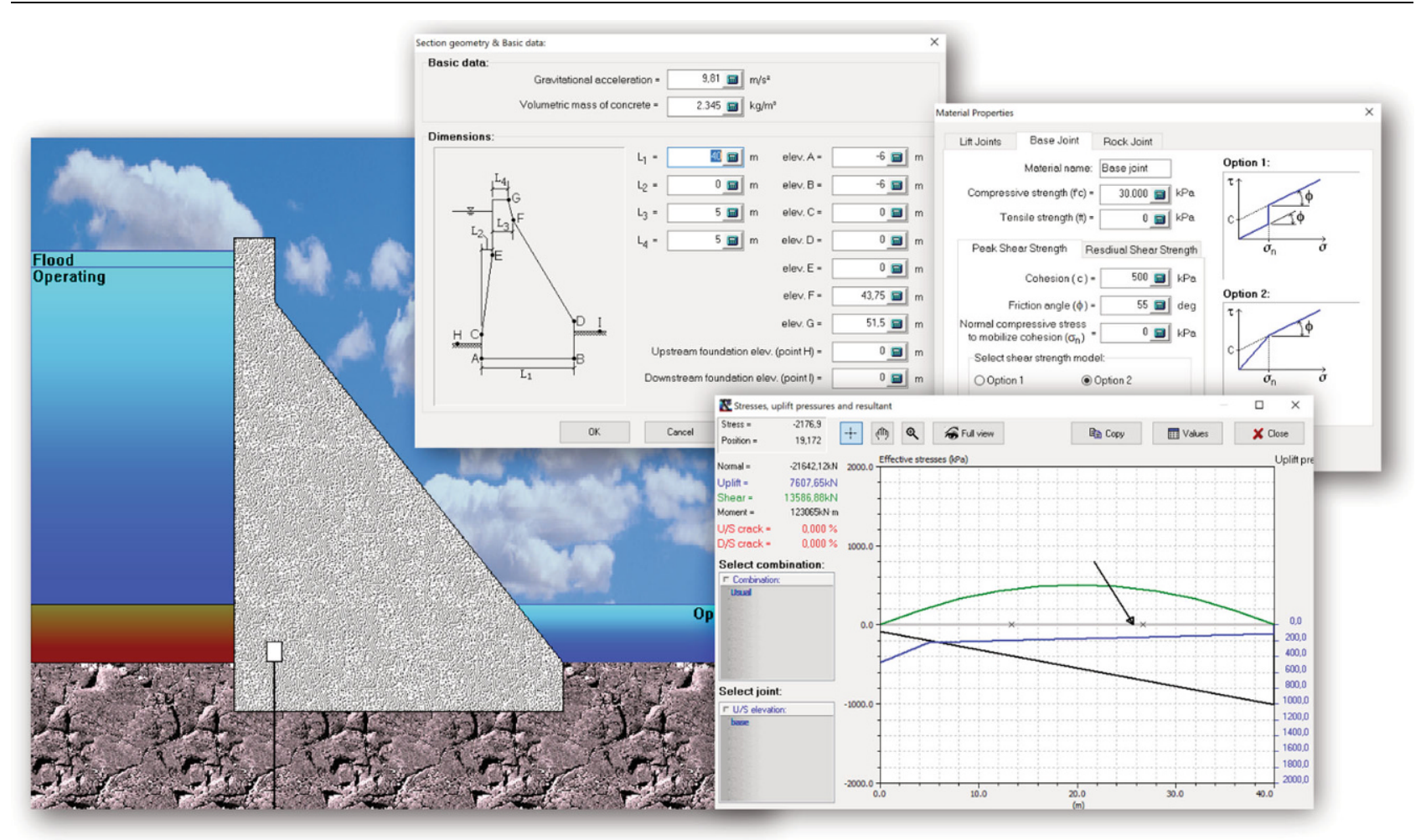

Abb. 1 Grafische Benutzerumgebung CADAM2D

numerische Modellierung von Talsperren und der damit zusammenhängende Erfahrungsaustausch im Fokus stehen (Staudacher et al. 2018).

Numerische Modelle kommen aus den bereits genannten Gründen auch bei der Berechnung von Gewichtsmauern zum Einsatz. Bei diesem Staumauertyp erfolgt die Ableitung der einwirkenden Lasten in den Untergrund über die Kontaktfläche zwischen Mauerbeton und Felsuntergrund. Die Nachweisführung ist grundsätzlich mittels Handrechnung bzw. durch den Einsatz von Tabellenkalkulationsprogrammen möglich. Abgesehen davon stehen für komplexere Berechnungen neben kommerziell vertriebenen FE-Codes auch frei verfügbare, Open-Source-FE-Softwarepakete wie z.B. code_aster von Électricité de France (EDF) oder EAGD84 (Fenves und Chopra 1984), ein an der Universität Berkeley (Kalifornien) entwickelter Code für seismische Berechnungen von Gewichtsmauern zur Verfügung. Abschließend sei noch das Programm CADAM2D erwähnt, dessen Funktionen in diesem Beitrag vorgestellt werden.

\section{Computer Analysis of DAMs (CADAM2D)}

Computer Analysis of DAMs (CADAM2D) ist eine frei verfügbare Software zur Berechnung der Standsicherheit von Gewichtsmauern in 2D (siehe Abb. 1). Erstellt an der Bauingenieurfakultät der École Polytechnique Montréal in Kanada (Leclerc et al. 2003) steht das Programm aktuell in der Version 2.0.4 auch zur freien Verfügung (https://cadam3d. ca/downloads.html\#cadam2d). Durch die relativ einfache Handhabung und die flache Lernkurve ist das Programm vor allem in der Ausbildung einsetzbar und ermöglicht rasch Analysen für unterschiedliche Lasten und deren Kombinationen. Die folgende Auflistung soll einen generellen Überblick über die Funktionalität des Programms geben:

- Statische Analysen unter Berücksichtigung des Eigengewichts und zusätzlicher Punktmassen sowie der grundlegenden äußeren Einwirkungen wie Wasserlasten, Sedimentlasten und Auftrieb. Zusätzlich können Lastfälle wie Eisdruck, Lasten zufolge Schwemmgut bzw. Überströmen des Bauwerks und Kräfte zufolge Vorspannankern eingerechnet werden. Die Evaluierung der Standsicherheit nach einem Erdbeben erfolgt mit- tels abgeminderter Scherwiderstände und adaptierter Wasserdruckverteilungen in den Fugen.

- Pseudo-statische Erdbebenanalysen, bei denen neben erwähnten Einwirkungen auch die Massenträgheitskräfte und hydrodynamischen Effekte (addierte Massen nach Westergaard 1933) einbezogen werden.

- Alternativ zur pseudo-statischen Methode ist eine vereinfachte Berechnung nach der Antwortspektrenmethode (pseudo-dynamische Erdbebenanalyse) möglich. Hierbei werden die komplexen Verhältnisse der Interaktionen zwischen Bauwerk, Untergrund und Stausee berücksichtigt.

- Probabilistische Sicherheitsanalyse (Monte-Carlo-Simulation) für statische und seismische Situationen zur Ermittlung der Versagenswahrscheinlichkeit unter Berücksichtigung von Unsicherheiten auf Lastund Widerstandsseite.

- Sensitivitätsanalyse durch inkrementelle Erhöhung äußerer Einwirkungen auf das Bauwerk, um die Entwicklung sicherheitsrelevanter Parameter wie z.B. Gleitsicherheit oder Länge der geöffneten Fuge zu berechnen. 


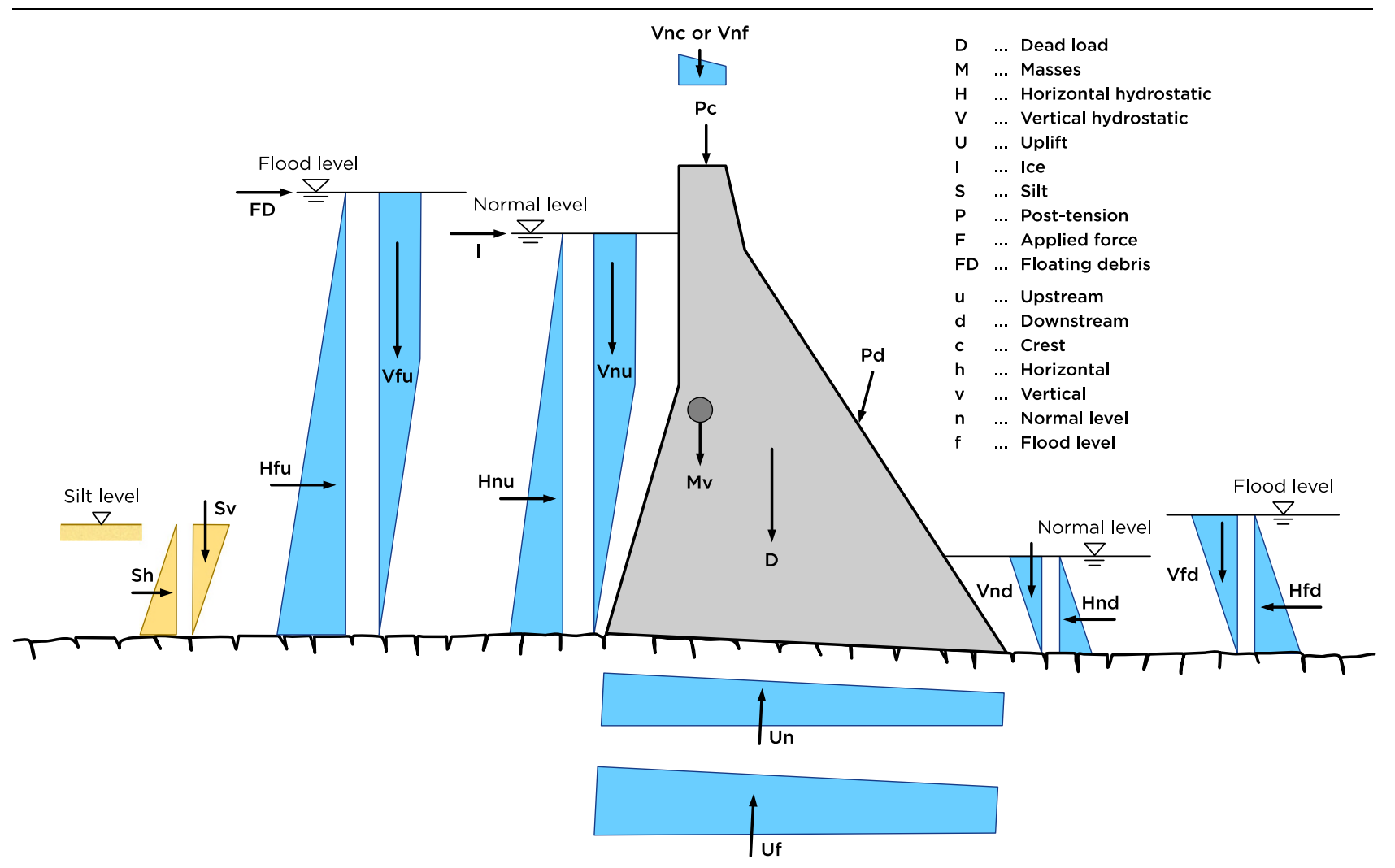

Abb. 2 Statische Lastfälle. (Mod. nach Leclerc und Léger 2019)

\subsection{Methodik zur Ermittlung der Bauwerksstabilität}

Grundsätzlich können mit dieser Software Gleit- und Kippsicherheitsfaktor und Auftriebssicherheit in der Sohlfuge und den Arbeitsfugen für die genannten Lastfälle und deren Kombinationen berechnet und dargestellt werden. Hierfür wird anhand der "Gravity Method“ (z. B. USACE 1995; USBR 1987) in den zu untersuchenden Fugen das Kräftegleichgewicht am ebenen und starren Körper bestimmt. Im nächsten Schritt erfolgt die Berechnung der Spannungsverteilung mit den auf die Fuge wirkenden Schnittkräften mithilfe der Balkentheorie, wobei auch ein Klaffen der Fugen zugelassen werden kann.

Die Nachweise in der jeweiligen Fuge des zu untersuchenden Querschnitts werden unter der Annahme durchgeführt, dass die Lasten ausschließlich über die Fuge abgetragen werden. Somit wird die Mitwirkung benachbarter Mauerblöcke, z.B. durch verpresste oder mit Schubverzahnung versehene Blockfugen, nicht berücksichtigt. Zusätzlich werden weitere Vereinfachungen wie homogene Materialeigenschaften des untersuchten Mauerblocks und konstante Scherwiderstände über die gesamte Länge der Fuge vorausgesetzt. In CADAM2D können die Nachweise neben horizontal ausgerichteten Fugen auch für geneigte Fugen (Richtung Ober- oder Unterwasser) relativ einfach und schnell erbracht werden.

\subsubsection{Berechnung der Spannungsverteilung}

Die Spannungen (Normalspannungen) an den ober- und unterwasserseitigen Randpunkten der jeweiligen Fuge werden mit der Balkentheorie nach Bernoulli unter Berücksichtigung der einwirkenden Lastresultierenden berechnet und für die Nachweisführung herangezogen. Ausgehend von den Randpunkten wird über den Querschnitt eine lineare Spannungsverteilung angenommen.

Wird ein Öffnen der Fuge in der Berechnung zugelassen, kann die Länge der Klaffung anhand der effektiven Spannungsverteilung am ungerissenen Querschnittsanteil unter Berücksichtigung der zulässigen Zugfestigkeit der Fuge berechnet werden. Entsprechend der gerissenen Länge wird der mit dem Stauspiegel korrespondierende Wasser- druck bis zur Rissspitze als konstant angenommen. Dieses nicht-lineare Problem wird durch Iteration gelöst, bis der Gleichgewichtszustand (oder ein Abbruchkriterium) erreicht ist. Das Ausmaß der Fugenöffnung hängt primär von den zulässigen Zugfestigkeiten der Fuge im ungerissenen bzw. gerissenen Zustand ab. Zusätzlich spielen die Verteilung sowie die Änderung des Wasserdrucks (gegebenenfalls Berücksichtigung der Drainage) in der Fuge eine Rolle.

Für den speziellen Fall einer horizontalen Fuge lassen sich die effektiven Normalspannungen am intakten Anteil des untersuchten Querschnitts wie folgt angeben:

$$
\sigma_{n}=\frac{\sum F_{v}}{A} \pm \frac{\Sigma M \cdot y}{I}
$$

$\Sigma F_{v} \ldots$ resultierende Vertikalkraft inkl. Auftrieb

A... Fläche des ungerissenen Querschnitts

$\Sigma M \ldots$.. Summe der Momente um den Mittelpunkt der intakten Fläche inkl. Auftrieb

I . . Flächenträgheitsmoment des ungerissenen Querschnitts 


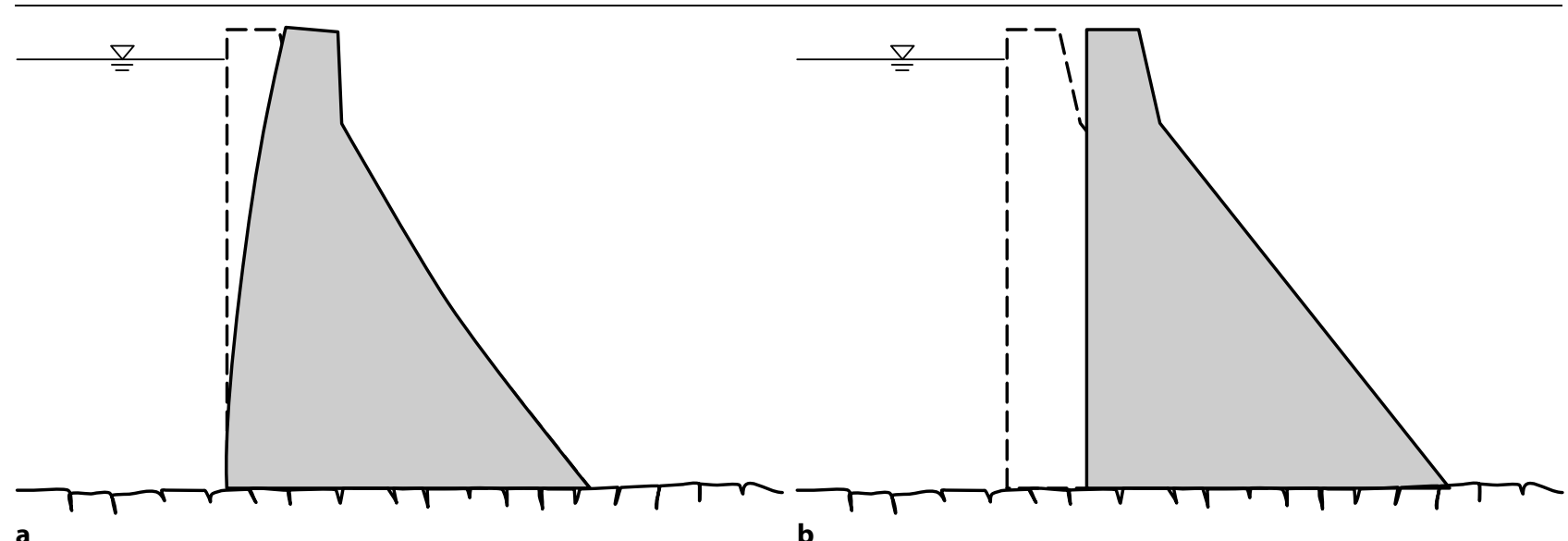

a

Abb. 3 Beschleunigung der Talsperre in ihrer ersten Eigenform (a), horizontale Beschleunigung des starren Sperrenkörpers (b). (Mod. nach Løkke und Chopra 2015)

$y \quad$ Abstand vom Mittelpunkt des Querschnitts bis zum Rand, in dem die Spannung berechnet wird (Randabstand)

\subsubsection{Gleitsicherheit}

Der Berechnung des Sicherheitsfaktors erfolgt mit den effektiven Normalkräften am ungerissenen Querschnittsanteil der Fuge, nachfolgend für eine horizontale Fuge dargestellt:

$$
S F=\frac{\left(\Sigma \overline{F_{v}}-U\right) \cdot \tan (\varphi)+c \cdot A_{c}}{\Sigma F_{h}}
$$

$\Sigma \overline{F_{v}} \ldots$ resultierende Vertikalkraft ohne Auftriebskraft

$U \ldots \quad$ Auftriebskraft

$\varphi \ldots \quad$ Reibungswinkel

$c . . \quad$ Kohäsion

$A_{c} \ldots$ überdrückter Anteil des Querschnitts

$\Sigma F_{h} \ldots$ resultierende Horizontalkraft

Optional kann für den Nachweis in der Aufstandsfläche ein zusätzlicher Scherwiderstand eines Felskeils im Unterwasser der Staumauer eingerechnet werden.

\subsubsection{Nachweise der Lagesicherheit gegen Kippen und Aufschwimmen}

Der Nachweis bezüglich Kippen erfolgt einerseits durch die Berechnung der Lage der Lastresultierenden in der untersuchten Trennfläche und andererseits durch Ermittlung der Kippsicherheit als Quotient aus rückhaltenden und treibenden Momenten um die ober- oder unterwasserseitigen Randpunkte der Fuge.

Die Auftriebssicherheit für den Anteil des Blocks über der betrachteten Fuge wird als Verhältnis aus der Summe der Vertikalkräfte ohne Auftrieb zur resultierenden Auftriebskraft gebildet.

\subsection{Statische Einwirkungen}

Wie eingangs bereits erwähnt, lassen sich alle gängigen und relevanten einwirkenden Lasten einbeziehen (siehe Abb. 2) und mit Teilsicherheitsfaktoren in verschiedenen Bemessungssituationen miteinander kombinieren. Die Auftriebsverteilung in den Fugen ist anhand der verschiedenen Ansätze lt. den internationalen Richtlinien wie z.B. USACE (1995) oder USBR (1987) möglich.

\subsection{Berechnungsmethoden für seismische Lastsituationen}

Seismische Einwirkungen werden durch Trägheitskräfte, welche zusätzlich auf das Bauwerk wirken, anhand des quasi-statischen Ansatzes einkalkuliert.Im Gegensatz dazu stehen Methoden zur Verfügung, welche die dynamischen Eigenschaften der Struktur berücksichtigen (z.B. modale Superposition oder Zeitreihenanalyse).

In CADAM2D sind zwei quasi-statische Ansätze zur Analyse seismischer Einwirkungen implementiert. Beim pseudo-statischen Ansatz werden die Trägheitskräfte mit der Maximalbeschleunigung einer Zeitreihe (engl. peak-ground-acceleration, kurz PGA) ermittelt. Der zweite Ansatz beruht auf der Antwortspektrenmethode, mit der die Beschleunigungen zur Berechnung der Trägheitskräfte anhand der dynamischen Eigenschaften (Eigenfrequenzen) des Systems bestimmt werden (siehe Abb. 3).

Wird eine nichtlineare statische Analyse durchgeführt, kann das Ergebnis als Initialzustand bei beiden genannten Ansätzen zur Erdbebenberechnung verwendet werden. Je nach Intensität und Wirkungsrichtung der Erdbebenbeanspruchung wird die Länge der Klaffung der Fuge neu berechnet. Hierbei wird die Standsicherheitsanalyse in zwei Abschnitten durchgeführt:

- Spannungsberechnung unter Annahme der Maximalbeschleunigung zur Berechnung der Klaffung, welche in die nachfolgenden Standsicherheitsanalysen Eingang findet, und

- Stabilitätsberechnung mit der abgeminderten Maximalbeschleunigung oder auch effektiven Beschleunigung. In Österreich wird lt. Erdbebenrichtlinie, Band 3 (Staubeckenkommission 1996) die Maximalbeschleunigung um $30 \%$ abgemindert.

\subsubsection{Pseudo-statischer Ansatz}

Diese Berechnung stellt die einfachste Art der Erdbebenanalyse dar, in der zusätzlich zu den statischen Kräften noch die Trägheitskräfte aufgrund der beschleunigten Massen mitberücksichtigt werden (siehe Abb. 4). Die im Erdbebenfall auftretende Interaktion von Talsperre (Fluid-Struktur-Interaktion, kurz FSI) und Stausee wird mit der Methode der addierten Massen nach Westergaard (1933) berücksichtigt. Die quasi-statische Analyse fußt auf der Annahme, dass die Staumauer als star- 


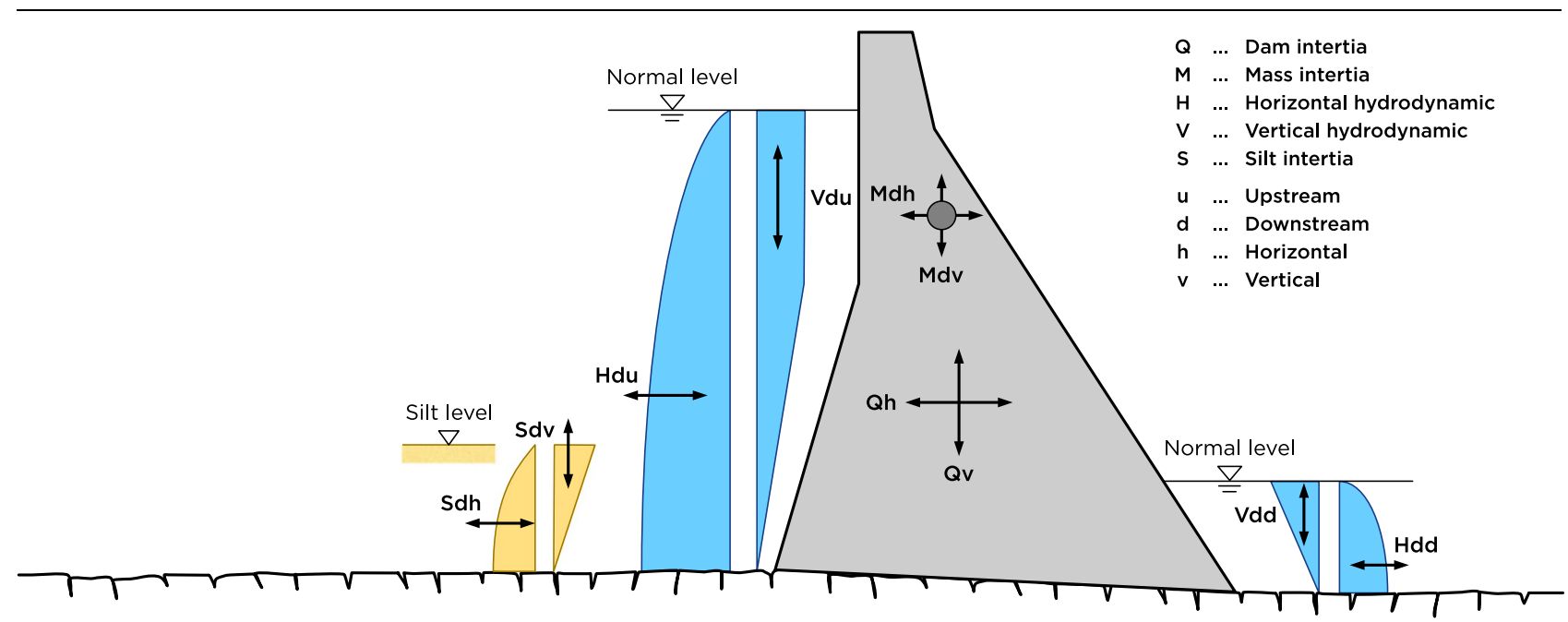

Abb. 4 Zusätzliche Trägheitskräfte im pseudo-statischen Ansatz. (Mod. nach Leclerc und Léger 2019)

rer Körper auf einem ebenso starren Fundament gegründet ist und es keine Amplifikation der Beschleunigung über die Mauerhöhe gibt. Die Trägheitskräfte werden mit der Maximalbeschleunigung (PGA) und der effektiven Beschleunigung berechnet.

\subsubsection{Pseudo-dynamischer Ansatz}

Prinzipiell ähneln sich die pseudo-statische und pseudo-dynamische Methode mit dem Unterschied, dass bei der pseudo-dynamischen Analyse ein elastisches System vorausgesetzt wird. Die Trägheitskräfte basieren auf spektralen Beschleunigungen, welche wiederum mittels Antwortspektren ermittelt werden. Anhand der modalen Superposition wird die maximale Systemantwort generiert, wodurch die Verstärkung der Basisbeschleunigung über die Höhe der Staumauer berücksichtigt wird (siehe Abb. 5).

Fenves und Chopra (1985a, 1985b) haben eine vereinfachte Methode zur Analyse von Gewichtsmauern postuliert. Dabei wird in CADAM2D die Geometrie der Mauer im zu untersuchenden Querschnitt auf starrem Untergrund auf einen äquivalenten Einmassenschwinger (EMS) reduziert und dessen erste Eigenfrequenz ermittelt. Durch Kombination der elastischen Eigenschaften von Beton und Felsuntergrund, zusammen mit dem Absorptionsverhalten von Druckwellen am Reservoirboden infolge von Sedimentationen, ist es möglich, anhand der von Fenves und Chopra durchgeführten Untersuchungen die Dämpfung des Gesamtsystems zu ermitteln. Wenn die
Dämpfung des Gesamtsystems (Staumauer, Untergrund und Reservoir) bekannt ist, lässt sich die gedämpfte (verlängerte) Periode des EMS berechnen. Nun sind die Periode und Dämpfungszahl bekannt und die zur Bemessung benötigte Beschleunigung wird im Bemessungsantwortspektrum abgelesen und zur Fortführung der Berechnung in CADAM2D eingegeben.

Das ursprüngliche Konzept wurde von Fenves und Chopra (1987) auf die höheren Eigenformen erweitert und ist auch in CADAM2D implementiert. Eine weitere Parameterstudie bezüglich der Dämpfung des Gesamtsystems (Løkke und Chopra 2013) erweiterte den Datenbestand und ist seit dem Jahr 2014 ebenfalls in CADAM2D integriert.

\subsection{Probabilistische Sicherheitsanalyse zur Ermittlung der \\ Versagenswahrscheinlichkeit}

Unter Umständen können sich Eingabewerte wie Materialparameter (Widerstände) und Einwirkungen im Vorfeld, im Zuge der Planung oder während des Betriebs der Anlage nicht exakt erfasst werden und sind daher mit einer gewissen Unsicherheit behaftet. CADAM2D stellt ein Modul zur Berechnung der Versagenswahrscheinlichkeit zur Verfügung, in dem die erwähnten Parameter als Zufallsvariablen angenommen werden und deren Verteilung mittels unterschiedlicher Dichtefunktionen möglich ist.

Die Lösung dieser Zufallsexperimente erfolgt mit Hilfe der Monte-CarloSimulation für eine große Anzahl von Experimenten $(<250.000)$. Dies ermög- licht die Berechnung der Versagenswahrscheinlichkeit durch statistische Auswertung dieser Zufallsexperimente für einen gewählten Versagensmechanismus (Gleiten oder Kippen).

\subsection{Sensitivitätsanalysen}

Ist eine Aussage über die Entwicklung einer Variablen (z.B. Gleitsicherheitsfaktor, Länge der Klaffung) in Abhängigkeit einer Einwirkung von Interesse, so lässt sich dies mit CADAM2D automatisiert bewerkstelligen. Mit dieser Analyse wird eine gewählte Einwirkung, in einer bestimmten Lastfallkombination (Bemessungssituation), schrittweise innerhalb eines definierten Wertebereichs erhöht und für jedes Inkrement die Spannungsverteilung sowie die für die Sicherheitsbeurteilung relevanten Ausgabeparameter in einer ausgewählten Fuge berechnet. So lässt sich der Einfluss auf den Gleitsicherheitsfaktor durch Variation der Drainageeffektivität darstellen (siehe Abb. 6).

Folgende Einwirkungen lassen sich mit der inkrementellen Analyse variieren:

- Wasserspiegel im Oberwasser bei Stauziel,

- Wasserspiegel im Oberwasser in der Hochwassersituation (Überstau),

- horizontale Komponente der Maximalbeschleunigung im Erdbebenfall,

- Eislast,

- Lasten aus Vorspannankern und

- Effektivität der Drainage. 


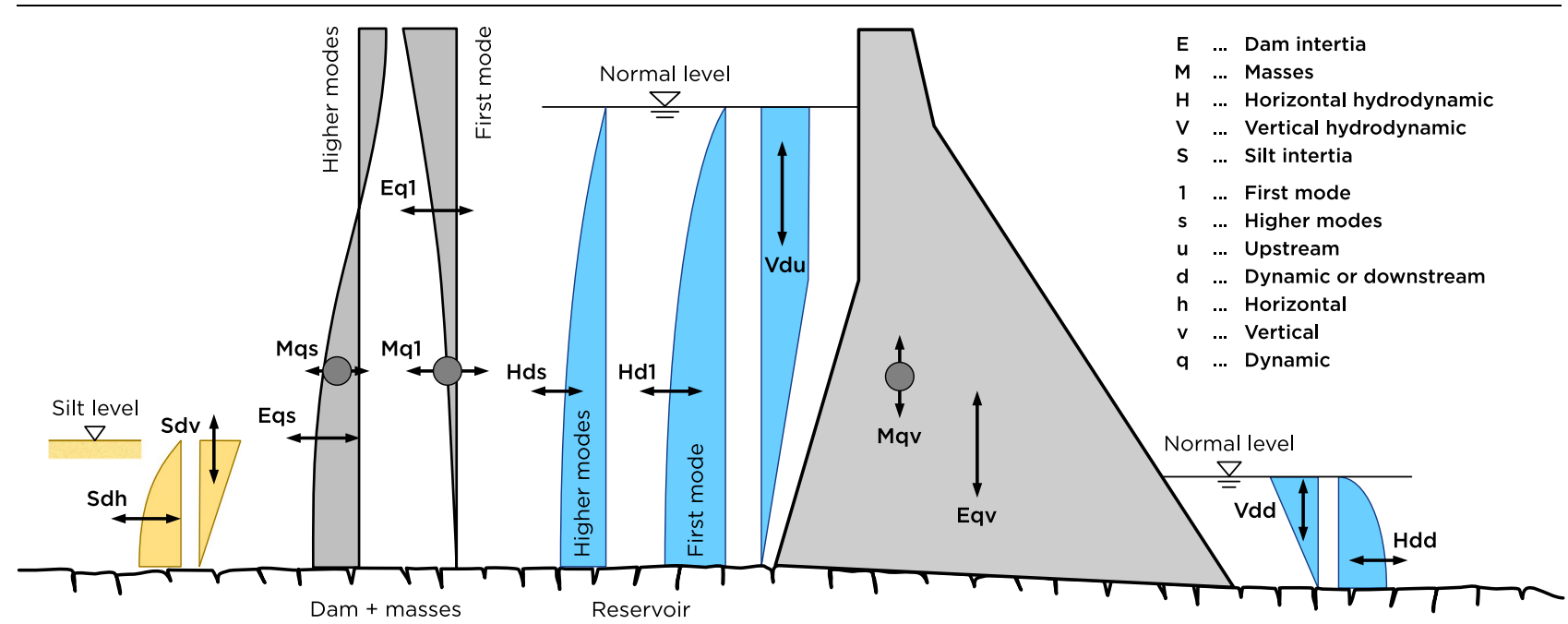

Abb. 5 Zusätzliche Trägheitskräfte im pseudo-dynamischen Ansatz. (Mod. nach Leclerc und Léger 2019)
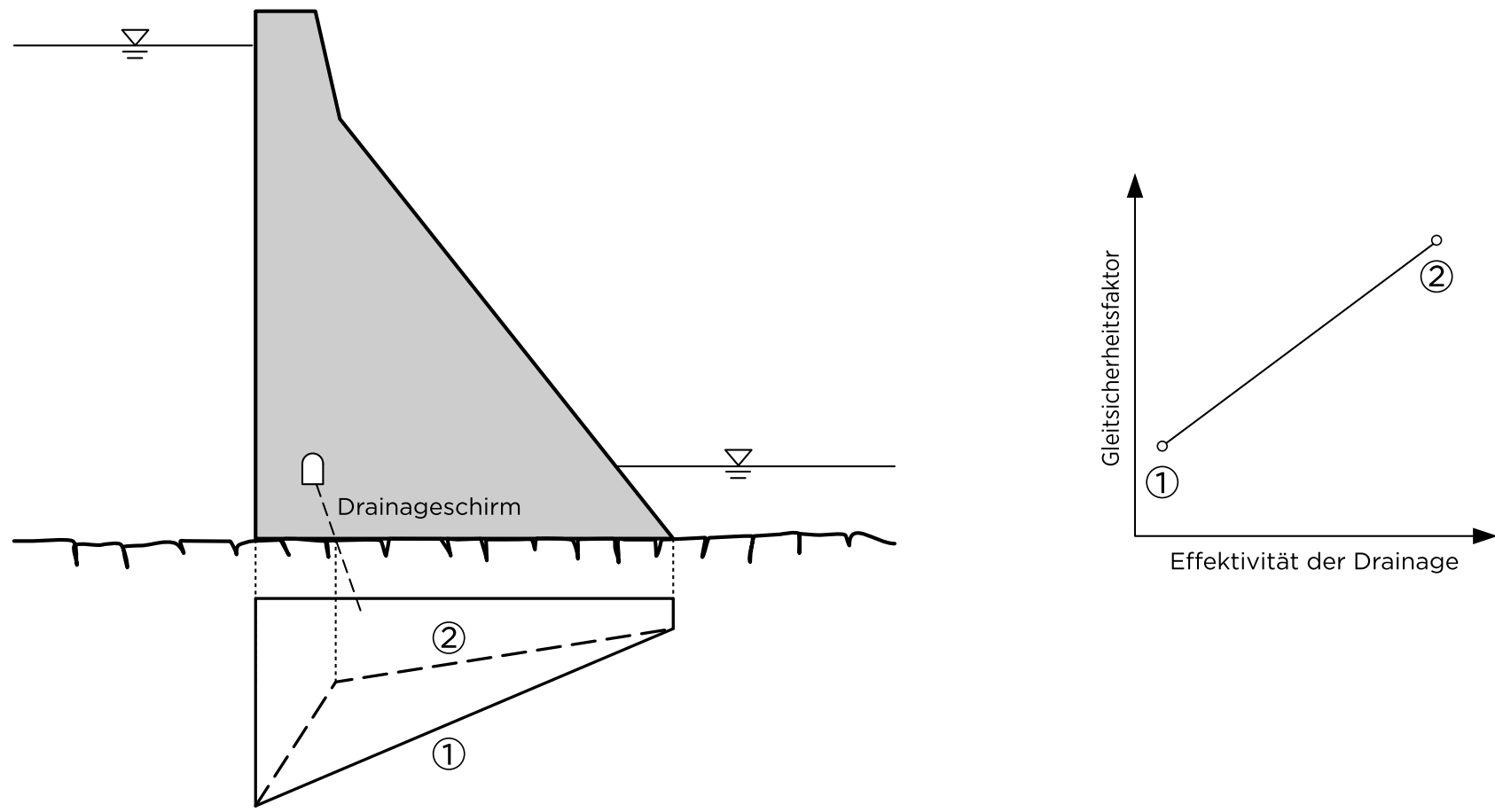

Abb. 6 Einfluss der Drainageeffektivität auf den Gleitsicherheitsfaktor in der Aufstandsfläche (schematische Darstellung)

\section{Anwendung in Österreich - Richtlinie für Betonsperren}

Seit 2016 sind die von der Staubeckenkommission (2016) erarbeiteten und veröffentlichen Richtlinien für den Nachweis der Tragsicherheit von Betonsperren in Kraft (siehe auch Obernhuber 2018). Die Beurteilung der Tragsicherheit von Gewichtsmauern kann mit CADAM2D entsprechend den Anforderungen in der österreichischen Richtlinie durchgeführt werden. Bei der Definition des Problems in CADAM2D ist darauf zu achten, dass die Designwerte der Scherfestigen (Reibungswinkel und Kohäsion) eingegeben werden, denn für diese Parameter ist keine Eingabe der Teilsicherheitsfaktoren vorgesehen. Eine richtlinienkonforme Reduktion der Auftriebswasserdrücke erfolgt durch Berücksichtigung des Ansatzes u. a. nach USACE (1995).

In Österreich entfällt der Kippnachweis für Gewichtsmauern, wenn in den Arbeitsfugen bzw. der Aufstandsfläche die zulässigen Klaffungen lt. Richtlinie eingehalten werden. Grundsätzlich ist
Kippen bei Gewichtsmauern mit gut proportioniertem Grunddreieck nicht relevant. Der Vollständigkeit halber wurde dieser Versagensmechanismus aber angeführt, da mit CADAM2D auch Strukturen untersucht werden können, deren Grundgeometrie sich von jener der Gewichtsmauern unterscheidet und somit Kippen von Relevanz sein kann.

Funding Open access funding provided by Graz University of Technology. 
Open Access Dieser Artikel wird unter der Creative Commons Namensnennung 4.0 International Lizenz veröffentlicht, welche die Nutzung, Vervielfältigung, Bearbeitung, Verbreitung und Wiedergabe in jeglichem Medium und Format erlaubt, sofern Sie den/die ursprünglichen Autor(en) und die Quelle ordnungsgemäß nennen, einen Link zur Creative Commons Lizenz beifügen und

Literatur angeben, ob Änderungen vorgenommen wurden.

Die in diesem Artikel enthaltenen Bilder und sonstiges Drittmaterial unterliegen ebenfalls der genannten Creative Commons Lizenz, sofern sich aus der Abbildungslegende nichts anderes ergibt. Sofern das betreffende Material nicht unter der genannten Creative Commons Lizenz steht und die betref- fende Handlung nicht nach gesetzlichen Vorschriften erlaubt ist, ist für die oben aufgeführten Weiterverwendungen des Materials die Einwilligung des jeweiligen Rechteinhabers einzuholen.

Weitere Details zur Lizenz entnehmen Sie bitte der Lizenzinformation auf http://creativecommons.org/licenses/ by/4.0/deed.de.
Fenves, G., Chopra, A.K. (1984): EAGD-84: A computer program for earthquake analysis of concrete gravity dams. Earthquake Engineering Research Center, University of California, Berkeley, California

Fenves, G., Chopra, A.K. (1985a): Simplified Earthquake Analysis of Concrete Gravity Dams: Separate Hydrodynamic and Foundation Interaction Effects. Journal of Engineering Mechanics 111:715-735. https://doi.org/10.1061/ (ASCE) 0733-9399(1985) 111:6(715)

Fenves, G., Chopra, A.K. (1985b): Simplified Earthquake Analysis of Concrete Gravity Dams: Combined Hydrodynamic and Foundation Interaction Effects. Journal of Engineering Mechanics 111:736-756. https://doi.org/10.1061/ (ASCE) 0733-9399(1985) 111:6(736)

Fenves, G., Chopra, A.K. (1987): Simplified Earthquake Analysis of Concrete Gravity Dams. Journal of Structural Engineering 113:1688-1708. https://doi.org/10.1061/(ASCE)0733-9445(1987) 113:8(1688)

Leclerc, M., Léger, P. (2019): CADAM2D User's Manual-Version 2.0.4, October 2019. Department of Civil, Geological and Mining Engineering Polytechnique Montréal, Montréal (Québec).

Leclerc, M., Léger, P., Tinawi, R. (2003): Computer aided stability analysis of gravity damsCADAM. Advances in Engineering Software 34:403-420. https://doi.org/10.1016/S09659978(03)00040-1
Løkke A, Chopra AK (2013): Response spectrum analysis of concrete gravity dams including dam-water-foundation interaction. PEER Report 2013/17, Pacific Earthquake Engineering Research Center, University of California, Berkeley, California.

Løkke, A., Chopra, A.K. (2015): Response Spectrum Analysis of Concrete Gravity Dams Including Dam-Water-Foundation Interaction. Journal of Structural Engineering 141:04014202. https://doi.org/10.1061/(ASCE)ST.1943-541X. 0001172

Obernhuber P (2018): Richtlinien für den Nachweis der Tragsicherheit von Betonsperren. Österr Wasser- und Abfallw 70: 388-396. https:// doi.org/10.1007/s00506-018-0491-7

Staubeckenkommission (1996): Erdbebenberechnung von Talsperren, Band 3. Staubeckenkommission, Bundesministerium für Landwirtschaft, Regionen und Tourismus, Wien. Staubeckenkommission (2016): Richtlinie zum Nachweis der Tragsicherheit von Betonsperren. Staubeckenkommission, Bundesministerium für Landwirtschaft, Regionen und Tourismus, Wien.

Staudacher, E., Zenz, G. (2017): Static and seismic assessment of an arch gravity dam. In: Malm et al. (Hrsg) Proceedings of the 14th ICOLD International Benchmark Workshop on Numerical Analysis of Dams, KTH Civil and Architectural Engineering, Stockholm. http://urn.kb.se/ resolve?urn=urn:nbn:se:kth:diva-223593. Gesehen am 15.04.2020

Staudacher, E., Zenz, G. (2019): 2D Seismic analysis of Pine Flat concrete gravity dam including mass of foundation-approach with infinite elements. In: Sterpi D. et al. (Hrsg) Proceedings of the 15th ICOLD Benchmark Workshop on Numerical Analysis of Dams, geplante Veröffentlichung im Jahr 2020, bei Springer Nature Switzerland AG.

Staudacher, E., Shahriari, S., Zenz, G. (2018): Numerische Modelle - ein Standbein der Talsperrensicherheit. Österreichische Wasser- und Abfallw 70:397-405. https://doi.org/10.1007/ s00506-018-0492-6

USACE (US Army Corps of Engineers) (1995): Engineering and design: Gravity dam design. Report EM 1110-2-2000, Washington, DC

USBR (United States Bureau of Reclamation) (1987): Design of small dams. Denver, Colorado. Denver, Colorado

Westergaard, H.M. (1933): Water Pressures On Dams During Earthquakes. Transactions, ASCE, 98:418-433.

Hinweis des Verlags Der Verlag bleibt in Hinblick auf geografische Zuordnungen und Gebietsbezeichnungen in veröffentlichten Karten und Institutsadressen neutral. 